\title{
A note on identities in two variables for a class of monoids
}

\section{Enrique Salcido $^{1}$ and Emil Daniel Schwab ${ }^{2}$}

\author{
${ }^{1}$ MS student, Department of Mathematical Sciences, The University of Texas at El Paso \\ El Paso, Texas 79968, USA \\ e-mail: esalcido@miners.utep.edu \\ ${ }^{2}$ Department of Mathematical Sciences, The University of Texas at El Paso \\ El Paso, Texas 79968, USA \\ e-mail: eschwab@utep. edu
}

Received: 30 August 2019 Revised: 24 December $2019 \quad$ Accepted: 12 January 2020

\begin{abstract}
In this note we consider identities in the alphabet $X=\{x, y\}$. This note is selfcontained and the aim is to describe gradually the identities partition (with three parameters) of the free semigroup $X^{+}$for the class of monoids $B_{n}=\left\langle a, b \mid b a=b^{n}\right\rangle(n>0)$.
\end{abstract}

Keywords: Semigroup identities, Checking identities, Identities partition.

2010 Mathematics Subject Classification: 68R15, 08A50.

\section{Introduction and preliminaries}

Section 4 of the recent paper by Geroldinger and Schwab [2] is devoted to the study of non-unique factorizations in a class of non-commutative monoids $\left\{B_{n}\right\}_{n>1}$. The monoids $B_{n}, n \in \mathbb{N}$, where $\mathbb{N}$ denotes the set of nonnegative integers, are defined by the monoid presentation:

$$
B_{n}=\left\langle a, b \mid b a=b^{n}\right\rangle .
$$

The elements of $B_{n}$ are words of the form $a^{k} b^{m}$ for $k, m \in \mathbb{N}$ with the understanding that $a^{0}=b^{0}=1$. The monoid $B_{0}$ is the bicyclic monoid which plays a very important role in the structural theory of semigroups. The multiplication in $B_{0}$ is given by the rule 


$$
a^{k} b^{m} \cdot a^{r} b^{s}=\left\{\begin{array}{lll}
a^{k} b^{m-r+s} & \text { if } & m \geq r \\
a^{k-m+r} b^{s} & \text { if } & m<r .
\end{array}\right.
$$

If $n>0$, then the multiplication in $B_{n}$ is defined by:

$$
a^{k} b^{m} \cdot a^{r} b^{s}=\left\{\begin{array}{ccc}
a^{k+r} b^{s} & \text { if } & m=0 \\
a^{k} b^{m+(n-1) r+s} & \text { if } & m>0 .
\end{array}\right.
$$

The identity (see [1])

$$
\text { (I) } \quad \text { yxуxyyx } \approx \text { xyyxyxxyyx, }\left(x y^{2} x^{2} y x y^{2} x \approx x y^{2} x y x^{2} y^{2} x\right)
$$

called Adjan's identity, is the first known and the shortest nontrivial identity satisfied in the bicyclic monoid $B_{0}$. It is known that $(I)$ and the identity

$$
\text { xyyxxyyxxy } \approx \text { xyyxyxyxxy }\left(x y^{2} x^{2} y^{2} x^{2} y \approx x y^{2} x y x y x^{2} y\right)
$$

are the only identities in the alphabet $\{x, y\}$ of length 10 satisfied in the bicyclic monoid $B_{0}$.

Now, we present some notations, definitions and remarks that are used throughout this paper. If $X$ is a given finite set (called the set of alphabet), $x \in X$, and $v$ is a word whose letters belong to $X$ then $n_{x}(v)$ denotes the number of occurrences of $x$ in $v$, and $\ell(v)=\sum_{x \in X} n_{x}(v)$ is called the length of $v$. A pair of words $(v, w)$ is called balanced if $n_{x}(v)=n_{x}(w)$ for all $x \in X$. A mapping $\sigma: X \rightarrow S$, where $S$ is a semigroup, can be extended in a unique way to a homomorphism of $X^{+}$(the free semigroup generated by $X$ ) to $\mathrm{S}$, called a substitution by elements of $S$, denoted again by $\sigma$. An identity $v \approx w\left(v, w \in X^{+}\right)$for (or, satisfied in) the semigroup $S$ is a pair $(v, w) \in X^{+} \times X^{+}$such that $\sigma(v)=\sigma(w)$ for all substitutions by elements of $S$. An identity $v \approx w$ is called balanced if the pair of words $(v, w)$ is balanced. In this case $\ell(v)=\ell(w)$ is called the length of the identity $v \approx w$. If $v \approx w$ is an identity for $S$ then the identity $u v u^{\prime} \approx u w u^{\prime}$ satisfied in $S$, where $u, u^{\prime} \in X^{*}\left(X^{*}\right.$ being $X^{+}$adjoined by the empty word) is called a simple consequence of $v \approx w\left(u\right.$ and $u^{\prime}$ are called prefix and suffix, respectively, of the words $u v u^{\prime}$ and $u w u^{\prime}$ ). The relation $\approx$ is an equivalence relation on the set $X^{+}$. The set of its equivalence classes is the identities partition for $S$, denoted by $\mathcal{P}_{S}$.

In this note we will consider the case $X=\{x, y\}$ and $S=B_{n}$ with $n>0$. The problem is to give characterizations of identities in $B_{n}(n>0)$. Shneerson [5] solved it (even in case of an arbitrary finite set $X): v \approx w$ is an identity satisfied in $B_{n}(n>0)$ if and only if $v=u v^{\prime}$, $w=u w^{\prime}$, where $u$ is a prefix of $v$ of the smallest length that contains both variables $x$ and $y$, and the words $v^{\prime}$ and $w^{\prime}$ are equal in the free commutative monoid over the alphabet $X=\{x, y\}$. Pastijn [3] solved this problem if $n=0$ in all its generality (X being countable infinite set) in three distinct ways, and Shleifer [4] studied also (only if $X=\{x, y\}$ ) identities for $B_{0}$ and created such identities using computer assistance.

The purpose of this note is to reach the above Shneerson's characterization gradually, if $X=\{x, y\}$. It is straightforward to see that Theorems 3.1 and 3.2 of Section 3 express this fact. In preparation we used only a few identities (Section 2). 


\section{The identities $\left(A_{i, j}\right)$}

Since $B_{n}$ (for all $n \in \mathbb{N}$ ) contains a copy of the infinite cyclic semigroup, any identity $v \approx w$ for $B_{n}$ is balanced. From the multiplication defined in $B_{n}$ it follows that if $v \approx w$ is an identity satisfied in $B_{n}$ then the first letter of $v$ and $w$ coincide. We will consider identities with the first letter $x$; changing the two letters $x$ and $y$ between them in each of the words of the identity $v \approx w$ does not lead to a new identity in our convention. If $n>0$ then the right cancellation law holds in the set of all identities for $B_{n}$ because the monoid $B_{n}(n>0)$ is right cancellative

Proposition 2.1. For any positive integers $i \geq j$ and $n>0$,

$$
\left(A_{i, j}\right) \quad x y^{i+1} x \approx x y^{j} x y^{i-j+1} \quad\left(\left(A_{i, j}^{\prime}\right) \quad y x^{i+1} y \approx y x^{j} y x^{i-j+1}\right)
$$

is an identity satisfied in $B_{n}$.

Proof. To prove that $\left(A_{i, j}\right)$ is an identity for $B_{n}(n>0)$ we consider the substitution $y=a^{k} b^{m}$, $x=a^{r} b^{s}$. Then

$$
\text { (a) } \quad y^{2} x=\left\{\begin{array}{ccc}
a^{2 k+r} b^{s} & \text { if } & m=0 \\
a^{k} b^{2 m+(n-1) k+(n-1) r+s} & \text { if } & m>0
\end{array}\right.
$$

and

$$
\text { (b) } \quad y x y=\left\{\begin{array}{cll}
a^{2 k+r} & \text { if } & m=0 \text { and } s=0 \\
a^{k+r} b^{s+(n-1) k} & \text { if } & m=0 \text { and } s>0 \\
a^{k} b^{2 m+(n-1) k+(n-1) r+s} & \text { if } & m>0 .
\end{array}\right.
$$

Since $y^{2} x=y x y$ in the cases $m>0$ and $m=0=s$, we will consider hereinafter $m=0, s>0$. Then

$$
x y^{i+1} x=x y^{i-1}\left(y^{2} x\right)=a^{r} b^{s} a^{(i-1) k} a^{2 k+r} b^{s}=a^{r} b^{s} a^{(i+1) k+r} b^{s}=a^{r} b^{2 s+(n-1)[(i+1) k+r]},
$$

and

$$
\begin{gathered}
x y^{i} x y=x y^{i-1}(y x y)=a^{r} b^{s} a^{(i-1) k} a^{k+r} b^{s+(n-1) k}=a^{r} b^{s} a^{i k+r} b^{s+(n-1) k}= \\
a^{r} b^{2 s+(n-1)[(i+1) k+r]} .
\end{gathered}
$$

So, $\left(A_{i, j}\right)$ is an identity satisfied in $B_{n}(n>0)$ if $i=j$.

Now, the following sequence of identities satisfied in $B_{n}, n>0$,

$$
x y^{i+1} x \approx x y^{i} x y \approx x y^{i-1} x y^{2} \approx x y^{i-2} x y^{3} \approx \cdots \approx x y^{j} x y^{i-j+1}
$$

finishes the proof of the proposition.

It is easy to check that nontrivial identities for $B_{n}$ of length 2 and 3 do not exist. It follows that:

Corollary 2.1. For any $n>0$, the identity

$$
\left(A_{1,1}\right) \quad x y^{2} x \approx x y x y \quad\left(\left(A_{1,1}^{\prime}\right) \quad y x^{2} y \approx y x y x\right)
$$

is the shortest nontrivial identity satisfied in the monoid $B_{n}$. 
Remark 2.1. The Adjan identity (I) and the identity (II) are both satisfied in $B_{n}$ since they are simple consequences of $\left(A_{1,1}^{\prime}\right)$ if $n>0$ :

$$
\begin{aligned}
& \left(A_{1,1}^{\prime}\right) \Rightarrow \underline{x y} \overbrace{y x^{2} y} \underline{\underline{x y^{2} x}} \approx \underline{x y} \overbrace{y x y x} \underline{\underline{x y^{2} x}} \text { that is (I); } \\
& \left(A_{1,1}^{\prime}\right) \Rightarrow \underline{x y} \overbrace{y x^{2} y} \underline{\underline{y x^{2} y}} \approx x y \overbrace{y x y x} \underline{\underline{y x^{2} y}} \text { that is (II). }
\end{aligned}
$$

Remark 2.2. Example 4.4 of [3] sets that

$$
x y x x^{i} y x^{\ell-i} y^{k} x \approx x y x x^{j} y x^{\ell-j} y^{k} x \quad(0 \leq i<j<\ell \text { and } k \geq 1)
$$

is an identity for $B_{0}$ if and only if $(k+1)(i+1) \geq \ell+1 \geq 2(j+1)$. The problem gets a new look in the case $n>0$. Using $\left(A_{i, 1}^{\prime}\right)$ and $\left(A_{j, 1}^{\prime}\right)$ we obtain the following two identities satisfied in $B_{n}, n>0$ :

$$
x y x x^{i} y x^{\ell-i} y^{k} x \approx x y x y x^{\ell} y^{k} x \quad \text { and } \quad x y x x^{j} y x^{\ell-j} y^{k} x \approx x y x y x^{\ell} y^{k} x .
$$

So, $x y x x^{i} y x^{\ell-i} y^{k} x \approx x y x x^{j} y x^{\ell-j} y^{k} x$ is an identity for $B_{n}$ (if $n>0$ ) for any $i, j, k, \ell \in \mathbb{N}$ with $i, j \leq \ell$.

\section{Main results}

Unless otherwise indicated, we consider words $v$ (and identities) with $x$ the first letter and with $n_{y}(v)>0$ (that is, words $v$ of the form $v=x^{k} u$, where $u$ is non-empty and $y$ is the first letter of $u$ ). We say that a word of the form

$$
\text { (*) } \quad x^{\ell_{1}}(y x)^{\ell_{2}} z^{\ell_{3}} \quad\left(\text { where } z \in\{x, y\}, \ell_{1}>0 \text { and } \ell_{2}, \ell_{3} \geq 0\right)
$$

is a canonical form of the word $v$ (the words $(y x)^{\ell_{2}}$ and $z^{\ell_{3}}$ are the empty word if $\ell_{2}=0$ and $\ell_{3}=0$, respectively) if

$$
v \approx x^{\ell_{1}}(y x)^{\ell_{2}} z^{\ell_{3}}
$$

is an identity satisfied in $B_{n}, n>0\left(\ell_{2}\right.$ can be 0 only if $\ell_{3}>0$ and $z=y$ since $\left.n_{y}(v)>0\right)$.

Lemma 3.1. A canonical form of the word $v=x^{k} u$ (y being the first letter of $u$ ), is given by

$$
v \approx\left\{\begin{array}{lll}
x^{k}(y x)^{n_{x}(u)} y^{n_{y}(u)-n_{x}(u)} & \text { if } & n_{y}(u) \geq n_{x}(u) \\
x^{k}(y x)^{n_{y}(u)} x^{n_{x}(u)-n_{y}(u)} & \text { if } \quad n_{y}(u)<n_{x}(u)
\end{array} .\right.
$$

Proof. A sequence of identities obtained by using (from left to right) only the identities $\left(A_{i, 1}\right)$ and $\left(A_{i, 1}^{\prime}\right)$ (i.e., $x y^{i+1} x \approx x y x y^{i}$ and $\left.y x^{i+1} y \approx y x y x^{i}\right)$ leads us in the end to an identity for $B_{n}$ of the form

$$
v \approx x^{k} y x y x \cdots y x z^{m} \quad(m \geq 0)
$$

where $z \in\{x, y\}$. It is clear that if $n_{y}(u)>n_{x}(u)$ then $z=y$ and the number of occurrences of $(y x)$ is $n_{x}(u)$. If $n_{y}(u)=n_{x}(u)$ then the number of occurrences of $(y x)$ is also $n_{x}(u)$. Since any identity for $B_{n}$ is balanced, it follows that $m=n_{y}(u)-n_{x}(u)$. Now, if $n_{y}(u)<n_{x}(u)$ then $z=x$ and the number of occurrences of $(y x)$ is $n_{y}(u)$. Obviously in this case $m=n_{x}(u)-n_{y}(u)$. 
Theorem 3.1. Let $v$ and $w$ be two words in the alphabet $\{x, y\}, v=x^{k} u$ and $w=x^{k^{\prime}} u^{\prime}$ (y being the first letter of both words $u$ and $\left.u^{\prime}\right)$. Then the following statements are equivalent:

(i) $v \approx w$ is an identity satisfied in $B_{n}, n>0$;

(ii) $v$ and $w$ have the same canonical form;

(iii) $n_{x}(u)=n_{x}\left(u^{\prime}\right), n_{y}(u)=n_{y}\left(u^{\prime}\right)$ and $k=k^{\prime}$;

$(i v)(v, w)$ is balanced and $k=k^{\prime}$.

Proof. $(i) \Leftrightarrow(i i)$ If $v$ and $w$ have the same canonical form then obviously $v \approx w$ is an identity satisfied in $B_{n}$ if $n>0$.

Conversely, if $v \approx w$ is an identity for $B_{n}, n>0$, and $v \approx x^{\ell_{1}}(y x)^{\ell_{2}} z^{\ell_{3}}, w \approx x^{\ell_{1}^{\prime}}(y x)^{\ell_{2}^{\prime}} z^{\prime \ell_{3}^{\prime}}$, are two canonical forms of $v$ and $w$ respectively, then we will prove that the two canonical forms are the same, that is: (1) $\ell_{1}=\ell_{1}^{\prime}, \ell_{2}=\ell_{2}^{\prime}, \ell_{3}=\ell_{3}^{\prime}$, and (2) $z=z^{\prime}$ if $\ell_{3}=\ell_{3}^{\prime} \neq 0$.

Using the substitution $\sigma_{1,1}$ by elements of $B_{n}(n>0)$ defined by $x=a, y=b$,

$$
\sigma_{1,1}\left(x^{\ell_{1}}(y x)^{\ell_{2}} z^{\ell_{3}}\right)=a^{\ell_{1}} b^{n \ell_{2}} b^{\ell_{3}}=a^{\ell_{1}} b^{n \ell_{2}+\ell_{3}} \quad \text { if } z=y
$$

and

$$
\sigma_{1,1}\left(x^{\ell_{1}}(y x)^{\ell_{2}} z^{\ell_{3}}\right)=a^{\ell_{1}} b^{n \ell_{2}} a^{\ell_{3}}=a^{\ell_{1}} b^{n \ell_{2}+(n-1) \ell_{3}} \quad \text { if } z=x .
$$

Analogously,

$$
\sigma_{1,1}\left(x^{\ell_{1}^{\prime}}(y x)^{\ell_{2}^{\prime}} z^{\prime \ell_{3}^{\prime}}\right)=a^{\ell_{1}^{\prime}} b^{n \ell_{2}^{\prime}+\ell_{3}^{\prime}} \quad \text { if } z^{\prime}=y
$$

and

$$
\sigma_{1,1}\left(x^{\ell_{1}^{\prime}}(y x)^{\ell_{2}^{\prime}} z^{\ell_{3}^{\prime}}\right)=a^{\ell_{1}^{\prime}} b^{n \ell_{2}^{\prime}+(n-1) \ell_{3}^{\prime}} \text { if } z^{\prime}=x .
$$

It is clear that $\sigma_{1,1}\left(x^{\ell_{1}}(y x)^{\ell_{2}} z^{\ell_{3}}\right)=\sigma_{1,1}\left(x^{\ell_{1}^{\prime}}(y x)^{\ell_{2}^{\prime}} z^{\ell_{3}^{\prime}}\right)$ implies

$$
\ell_{1}=\ell_{1}^{\prime}
$$

Since any identity for $B_{n}$ is balanced, it follows that

$$
2 \ell_{2}+\ell_{3}=2 \ell_{2}^{\prime}+\ell_{3}^{\prime}
$$

The equality $\sigma_{1,1}\left(x^{\ell_{1}}(y x)^{\ell_{2}} z^{\ell_{3}}\right)=\sigma_{1,1}\left(x^{\ell_{1}^{\prime}}(y x)^{\ell_{2}^{\prime}} z^{\prime \ell_{3}^{\prime}}\right)$ implies also:

Case 1. $\left(z=z^{\prime}=y\right): n \ell_{2}+\ell_{3}=n \ell_{2}^{\prime}+\ell_{3}^{\prime}$, that is $(n-2)\left(\ell_{2}^{\prime}-\ell_{2}\right)=0$.

Case 2. $\left(z=z^{\prime}=x\right): n \ell_{2}+(n-1) \ell_{3}=n \ell_{2}^{\prime}+(n-1) \ell_{3}^{\prime}$, that is $(n-2)\left(\ell_{2}^{\prime}-\ell_{2}\right)=0$.

Case 3. $\left(z \neq z^{\prime}\right)$ : if $z=y$ and $z^{\prime}=x$ then $n \ell_{2}+\ell_{3}=n \ell_{2}^{\prime}+(n-1) \ell_{3}^{\prime}$ implies $2 n\left(\ell_{2}^{\prime}-\ell_{2}\right)=$ $2 \ell_{3}-2(n-1) \ell_{3}^{\prime}$ and so, $(n-2)\left(\ell_{3}+\ell_{3}^{\prime}\right)=0$; analogously if $z=x$ and $z^{\prime}=y$. Thus the hypothesis $z \neq z^{\prime}$ implies $\ell_{3}=\ell_{3}^{\prime}=0$ if $n \neq 2$, and therefore $z^{\ell_{3}}$ and $z^{\prime \ell_{3}^{\prime}}$ are the empty word. 
Since $\ell_{2}^{\prime}=\ell_{2}$ if and only if $\ell_{3}^{\prime}=\ell_{3}$ (any identity satisfied in $B_{n}$ is balanced), the conclusion is that $\ell_{2}^{\prime}=\ell_{2}, \ell_{3}^{\prime}=\ell_{3}$ if $n \neq 2$, and $z=z^{\prime}$ if $\ell_{3}=\ell_{3}^{\prime} \neq 0$ and $n \neq 2(n>0)$. The case $n=2$ will be discussed below.

Let $\sigma_{1,2}$ be the substitution by elements of $B_{2}$ defined by $x=a, y=b^{2}$. Then,

$$
\sigma_{1,2}\left(x^{\ell_{1}}(y x)^{\ell_{2}} z^{\ell_{3}}\right)=a^{\ell_{1}} b^{3 \ell_{2}} a^{\ell_{3}}=a^{\ell_{1}} b^{3 \ell_{2}+\ell_{3}} \text { if } z=x
$$

and

$$
\sigma_{1,2}\left(x^{\ell_{1}}(y x)^{\ell_{2}} z^{\ell_{3}}\right)=a^{\ell_{1}} b^{3 \ell_{2}} b^{2 \ell_{3}}=a^{\ell_{1}} b^{3 \ell_{2}+2 \ell_{3}} \text { if } z=y .
$$

Analogously,

$$
\sigma_{1,2}\left(x^{\ell_{1}^{\prime}}(y x)^{\ell_{2}^{\prime}} z^{\ell_{3}^{\prime}}\right)=a^{\ell_{1}^{\prime}} b^{3 \ell_{2}^{\prime}+\ell_{3}^{\prime}} \text { if } z^{\prime}=x
$$

and

$$
\sigma_{1,2}\left(x^{\ell_{1}^{\prime}}(y x)^{\ell_{2}^{\prime}} z^{\prime \ell_{3}^{\prime}}\right)=a^{\ell_{1}^{\prime}} b^{3 \ell_{2}^{\prime}+2 \ell_{3}^{\prime}} \text { if } z^{\prime}=y .
$$

Taking into account that $\ell_{1}=\ell_{1}^{\prime}$ and $2 \ell_{2}+\ell_{3}=2 \ell_{2}^{\prime}+\ell_{3}^{\prime}$, the equality $\sigma_{1,1}\left(x^{\ell_{1}}(y x)^{\ell_{2}} z^{\ell_{3}}\right)=$ $\sigma_{1,1}\left(x^{\ell_{1}^{\prime}}(y x)^{\ell_{2}^{\prime}} z_{3}^{\ell_{3}^{\prime}}\right)$ implies:

Case 1. $\left(z=z^{\prime}=y\right): 3 \ell_{2}+\ell_{3}=3 \ell_{2}^{\prime}+\ell_{3}^{\prime} \Rightarrow \ell_{2}=\ell_{2}^{\prime}$ (and therefore $\left.\ell_{3}=\ell_{3}^{\prime}\right)$.

Case 2. $\left(z=z^{\prime}=x\right): 3 \ell_{2}+2 \ell_{3}=3 \ell_{2}^{\prime}+2 \ell_{3}^{\prime} \Rightarrow \ell_{2}=\ell_{2}^{\prime}$ (and therefore $\left.\ell_{3}=\ell_{3}^{\prime}\right)$.

Case 3. $\left(z \neq z^{\prime}\right)$ : if $z=y$ and $z^{\prime}=x$ then $3 \ell_{2}+\ell_{3}=3 \ell_{2}^{\prime}+2 \ell_{3}^{\prime} \Rightarrow \ell_{2}=\ell_{2}^{\prime}+\ell_{3}^{\prime}$ and so $2 \ell_{2}^{\prime}+\ell_{3}^{\prime}=2\left(\ell_{2}^{\prime}+\ell_{3}^{\prime}\right)+\ell_{3}$, that is $\ell_{3}^{\prime}+\ell_{3}=0$ and therefore $\ell_{3}^{\prime}=\ell_{3}=0$ (analogously if $z=x$ and $\left.z^{\prime}=y\right)$.

Thus, if $v \approx w$ is an identity for $B_{n}, n>0$, and $v \approx x^{\ell_{1}}(y x)^{\ell_{2}} z^{\ell_{3}}, w \approx x^{\ell_{1}^{\prime}}(y x)^{\ell_{2}^{\prime}} z^{\ell_{3}^{\prime}}$, are two canonical forms of $v$ and $w$ respectively, then the two canonical forms coincide.

(ii) $\Leftrightarrow$ (iii) follows from Lemma 3.1.

(iii) $\Leftrightarrow($ iv) holds obviously.

Remark 3.1. Given two different words $v$ and $w$, if $x^{k}(k>0)$ is the leftmost subword of the maximal length of both words $v$ and $w$ consisting of repetitions of $x, n_{y}(v)=n_{y}(w)=\ell>1$ and $n_{x}(v)-k=n_{x}(w)-k=m>0$ then, and only then, $v \approx w$ is a nontrivial identity for $B_{n}$ $(n>0)$. So, a triple of positive integers $(k, l, m), l>1$, determine a set of words and thus a set of nontrivial identities. For example, the triple of positive integers $(4,2,2)$ determine the set of words

$$
\left\{x^{4} y^{2} x^{2}, x^{4} y x^{2} y, x^{4} y x y x\right\}
$$

and the set of nontrivial identities

$$
\left\{x^{4} y^{2} x^{2} \approx x^{4} y x^{2} y, x^{4} y x^{2} y \approx x^{4} y x y x, x^{4} y^{2} x^{2} \approx x^{4} y x y x\right\}
$$

Taking into account all possible cases, we conclude that 
Theorem 3.2. The identities partition $\mathcal{P}_{B_{n}}(n>0)$ is given by

$$
\mathcal{P}_{B_{n}}=\left\{P_{k, l, m}\right\}_{k, l>0, m \geq 0} \cup\left\{P_{k, 0,0}\right\}_{k>0},
$$

where

$$
P_{k, l, m}=\left\{x^{k} u \mid \text { the first letter of } u \text { is } y, n_{y}(u)=l \text { and } n_{x}(u)=m\right\}
$$

if $k, l>0, m \geq 0$, and $P_{k, 0,0}(k>0)$ are the singletons $\left\{x^{k}\right\}$. The elements of this partition are finite sets and if $k, l>0, m \geq 0$, then

$$
\left|P_{k, l, m}\right|=\left(\begin{array}{c}
l+m-1 \\
l-1
\end{array}\right) .
$$

\section{References}

[1] Adjan, S.I. (1967). Defining relations and algorithmic problems for groups and semigroups, American Mathematical Society, Providence, (English translation of Proceeding of the Steklov Institute of Mathematics Vol. 85).

[2] Geroldinger, A. \& Schwab, E. D. (2018). Sets of lengths in atomic unit-cancellative finitely presented monoids, Colloq. Math., 151, 171-187.

[3] Pastijn, F. (2006). Polyhedral convex cones and the equational theory of the bicyclic semigroup, J. Aust. Math. Soc., 81, 63-96.

[4] Shleifer, F. G. (1990). Looking for identities on a bicyclic semigroup with computer assistance, Semigroup Forum, 41, 173-179.

[5] Shneerson, L. M. (1985). On the varieties generated by semigroups and monoids with one defining relation, Siberian Math. Journal 26, 202 (Abstract), Preprint VINITI No 3641-84, $1-32$. 